\title{
Wavelet Collocation Method for Optimal Control Problems
}

\author{
R. Dai · J. E. Cochran, Jr.
}

Published online: 15 May 2009

(C) The Author(s) 2009. This article is published with open access at Springerlink.com

\begin{abstract}
A Haar wavelet technique is discussed as a method for discretizing the nonlinear system equations for optimal control problems. The technique is used to transform the state and control variables into nonlinear programming (NLP) parameters at collocation points. A nonlinear programming solver can then be used to solve optimal control problems that are rather general in form. Here, general Bolza optimal control problems with state and control constraints are considered. Examples of two kinds of optimal control problems, continuous and discrete, are solved. The results are compared to those obtained by using other collocation methods.
\end{abstract}

Keywords Haar wavelet · Collocation · Discrete optimal control · Nonlinear programming

\section{Introduction}

Recently, wavelet theory has attracted considerable attention due to the advantages wavelets have over traditional Fourier transforms in accurately approximating functions that have discontinuities and sharp peaks. Wavelets have been applied in signal processing, multi-scale phenomena modeling and pattern recognition.

The Haar wavelet function was introduced by Alfred Haar in 1910 in the form of a regular pulse pair [1]. After that many other wavelet functions were generated and introduced. Those include the Shannon, Daubechies and Legendre wavelets. Among those forms, Haar wavelets have the simplest orthonormal series with compact support. These characteristics makes Haar wavelets good candidates for application to optimal control problems.

R. Dai · J.E. Cochran, Jr. (凶)

Department of Aerospace Engineering, Auburn University, Auburn, AL 36849, USA

e-mail: jcochran@eng.auburn.edu

R. Dai

e-mail: aviator_dai@hotmail.com 
The wavelet applications in dealing with dynamic system problems, especially in solving partial differential equations with two-point boundary value constraints have been discussed in many papers [2-5]. By transforming differential equations into algebraic equations, the solution may be found by determining the corresponding coefficients that satisfy the algebraic equations. Some efforts have been made to solve linear optimal control problems by using wavelet collocation [6-9]. But, when the system equations become complex and highly nonlinear, it is necessary to seek some useful tools to solve those kinds of problems. The nonlinear programming solver (NLPS) SNOPT $[10,11]$ which uses a Sequential Quadratic Programming algorithm to solve the nonlinear problems seems to be an efficient tool for this task. At each major iteration of the NLPS algorithm, the solver finds the search direction for the current nominally optimized points. This process is repeated until convergence occurs. Then the parameterized objective function can be optimized with system equation constraints satisfied.

The collocation methods developed to solve nonlinear optimal control problems generally fall into two categories, local collocation [12,13] and global orthogonal collocation [14-17]. In local collocation methods such as trapezoidal, HermiteSimpson and Runge-Kutta methods, the time interval considered is divided into a series of subintervals within which the integration rule must be satisfied. These local collocation methods were introduced in direct collocation and nonlinear programming (DCNLP) and have wide application [18-20]. In recent years, more attention has been focused on global orthogonal collocation methods such as Legendre, Chebyshev, Gauss method and some others. By expanding the state and control variables into piecewise-continuous polynomials, the derivative of the state variables can be approximated by combinations of these interpolating polynomials and their derivatives. Then, the objective function and system constraints are all converted into algebraic equations with unknown coefficients. The orthogonal collocation methods are generally better than local collocation methods in achieving fast convergence rate and high accuracy.

There are three major classes of orthogonal functions, the orthogonal polynomials, like Legendre, Chebyshev, etc., the sine-cosine functions in Fourier series and the constant basis function, like Haar, block-pulse, etc. The first two classes of orthogonal functions have been widely applied in collocation as mentioned above. But, apparently no attempts have been made to apply constant basis orthogonal functions in collocation. Thus, it is of interest to see how this new collocation method works.

In this paper, we first introduce the Haar wavelets theory and properties including the Haar wavelets basis and its integral operational matrix. Then we will assume that the control variables and derivatives of the state variables in the optimal control problems may be expressed in the form of Haar wavelets and unknown coefficients. The state variables can be calculated by using the Haar operational integration matrix. Therefore, all variables in the nonlinear system equations are expressed as series of the Haar family and its operational matrix. Finally, the task of finding the unknown parameters that optimize the designate performance while satisfying all constraints is performed by the NLPS. To demonstrate the applicability of this new collocation method, we consider two example optimal control problems, one with a smooth control and one with a nonsmooth control. 


\section{Haar Wavelets}

\subsection{Haar Functions}

The basic and simplest form of Haar wavelet is the Haar scaling function that appears in the form of a square wave over the interval $t \in[0,1)$ as expressed in (1) and illustrated in the first subplot of Fig. 1,

$$
\phi_{0}(t)= \begin{cases}1, & 0 \leq t<1, \\ 0, & \text { elsewhere }\end{cases}
$$

The above expression, called Haar father wavelet, is the zeroth level wavelet which has no displacement and dilation of unit magnitude. Correspondingly, there is a Haar mother wavelet to match the father wavelet which is described as

$$
\phi_{1}(t)= \begin{cases}1, & 0 \leq t<\frac{1}{2} \\ -1, & \frac{1}{2} \leq t<1 \\ 0, & \text { elsewhere }\end{cases}
$$

Fig. 1 Graph of Haar function
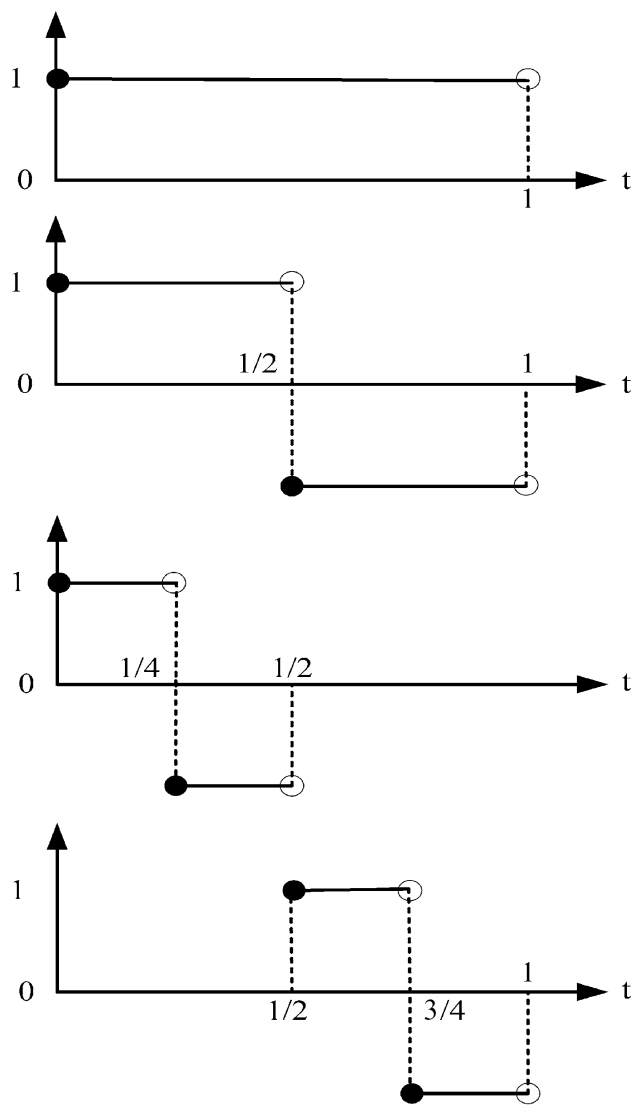
The Haar mother wavelet is the first level Haar wavelet and its graph is given in the second subplot of Fig. 1. This mother wavelet can also be written as the linear combination of the Haar scaling function with translation and compression to half of its original interval

$$
\phi_{1}(t)=\phi_{0}(2 t)-\phi_{0}(2 t-1) .
$$

Similarly, the other levels of wavelets can all be generated from $\phi_{1}(t)$ by the operations of translation and compression. For example, the third subplot in Fig. 1 is formed by compression $\phi_{1}(t)$ to left half of its original interval and the forth subplot is the same as the third one plus translating to the right side by $1 / 2$. In general, we can write out the Haar wavelet family as

$$
\phi_{i}(t)= \begin{cases}1, & t \in\left[\frac{k}{m}, \frac{k+0.5}{m}\right), \\ -1, & t \in\left[\frac{k+0.5}{m}, \frac{k+1}{m}\right) . \\ 0, & \text { elsewhere. }\end{cases}
$$

Here $m$ is the level of the wavelet, we assume the maximum level resolution is integer $J$, then $m$ equals to $2^{j}(j=0,1, \ldots, J)$; the translation parameter $k=$ $0,1, \ldots, m-1$. The series index number $i$ is defined by $m$ and $k$ and $i=m+k$. For any fixed level $m$, there are $m$ series of $\phi_{i}$ to fill the interval $[0,1)$ corresponding to that level and for a provided $J$, the index number $i$ can reach the maximum value $M=2^{J+1}$ when including all levels of wavelets. Each Haar wavelet is composed of a couple of constant steps of opposite sign during its subinterval and is zero elsewhere. Therefore, they have the following relationship:

$$
\int_{0}^{1} \phi_{i}(t) \phi_{l}(t) d t= \begin{cases}2^{-j}, & i=l=2^{j}+k, \\ 0, & i \neq l .\end{cases}
$$

This relationship shows that Haar wavelets are orthogonal to each other and therefore constitute an orthogonal basis. This allows us to transform any function square integrable on the interval time $[0,1)$ into Haar wavelets series.

\subsection{Function Approximation by Haar Wavelets}

We just pointed out that a square integrable function can be expressed in terms of Haar orthogonal basis on interval $\tau \in[0,1)$. However, before the procession to this transfer, it is necessary to unify the time interval. By using a linear transformation, the actual time $t$ can be expressed as a function of $\tau$ via

$$
t=\left[\left(t_{f}-t_{0}\right) \tau+t_{0}\right]
$$

where $t_{0}$ is the initial time and $t_{f}$ is the final time in a square integrable function $f(t)$. The objective is to write this $f(t)$ in form of wavelet expansion series with coefficients, that is,

$$
f(t)=\sum_{i=0}^{M-1} a_{i} \phi_{i}(t)=A^{T} \Psi_{M}(t),
$$


where the coefficient vector $A=\left[a_{0}, a_{1}, \ldots, a_{M-1}\right]^{T}$ and $\Psi_{M}(t)=\left[\phi_{0}(t), \phi_{1}(t), \ldots\right.$, $\left.\phi_{M-1}(t)\right]^{T}$. In the vector $A$, each coefficient $a_{i}$ is determined by

$$
a_{i}=2^{j} \int_{0}^{1} f(t) \phi_{i}(t) d t
$$

and it is expected to approximate the function $f(t)$ with minimum mean integral square error $\varepsilon$ defined as

$$
\varepsilon=\int_{0}^{1}\left(f(t)-A^{T} \Psi(t)\right) d t .
$$

Obviously, $\varepsilon$ should reduce when the level $M$ gets larger and it should go close to zero when $M$ approaches infinite. If we set all the collocation pick point $t_{s}$ at the middles of each wavelet, then $t_{s}$ is defined as

$$
t_{s}=(s-0.5) / M,
$$

where $s=1,2, \ldots, M$. With these chosen collocation points, the function is discretized into a series of nodes with equivalent distances. The vector $\Psi_{M}(t)$ can also be determined at those collocation points. Let the Haar matrix $H$ be the combination of $\Psi_{M}(t)$ at all the collocation points. Thus, we get

$$
\begin{aligned}
H_{M} & =\left[\Psi_{M}\left(t_{0}\right), \Psi_{M}\left(t_{1}\right), \ldots, \Psi_{M}\left(t_{M-1}\right)\right] \\
& =\left[\begin{array}{cccc}
\phi_{0}\left(t_{0}\right) & \phi_{0}\left(t_{1}\right) & \ldots & \phi_{0}\left(t_{M-1}\right) \\
\phi_{1}\left(t_{0}\right) & \phi_{1}\left(t_{1}\right) & \ldots & \phi_{1}\left(t_{M-1}\right) \\
\vdots & \vdots & \ddots & \vdots \\
\phi_{M-1}\left(t_{0}\right) & \phi_{M-1}\left(t_{1}\right) & \ldots & \phi_{M-1}\left(t_{M-1}\right)
\end{array}\right]_{M \times M}
\end{aligned}
$$

For example,

$$
H_{2}=\left[\Psi_{2}\left(t_{0}\right), \Psi_{2}\left(t_{1}\right)\right]=\left[\begin{array}{cc}
1 & 1 \\
0 & -1
\end{array}\right]
$$

and

$$
H_{4}=\left[\Psi_{4}\left(t_{0}\right), \Psi_{4}\left(t_{1}\right), \Psi_{4}\left(t_{2}\right), \Psi_{4}\left(t_{3}\right)\right]=\left[\begin{array}{cccc}
1 & 1 & 1 & 1 \\
1 & 1 & -1 & -1 \\
1 & -1 & 0 & 0 \\
0 & 0 & 1 & -1
\end{array}\right] .
$$

Therefore, the function $f(t)$ is approximated as $f\left(t_{s}\right) \approx C_{1 \times M}^{T} H_{M \times M}$.

\subsection{Haar Operational Integration Matrix}

In the solution of optimal control problems, we always need to deal with equations involving differentiation and integration. If the system function is expressed in Haar wavelets, the integration or differentiation operation of Haar series cannot be avoided. The differentiation of step waves will generate pulse signals which are difficult to 
handle, while the integration of step waves will result in constant slope functions which can be calculated by the following equation:

$$
\int_{0}^{1}\left(\Psi\left(t^{\prime}\right)\right) d t^{\prime}=P \Psi(t)
$$

where $P$ is the $M \times M$ operational integration matrix and is given by $\mathrm{Gu}$ and Jiang [21] for how to calculate this matrix,

$$
P_{M}==\left[\begin{array}{cc}
P_{M / 2} & -\frac{1}{2 M} H_{M / 2} \\
\frac{1}{2 M} H_{M / 2}^{-1} & 0
\end{array}\right]_{M \times M}, \quad P_{1}=\left[\begin{array}{l}
1 \\
2
\end{array}\right] .
$$

\section{Formulation of Optimal Control Problems}

The objective of an optimal control problem is to find the history of the control variable(s) that will maximize or minimize a given performance index while satisfying the system constraints. The system constraints include first-order, ordinary differential equations subject to initial and final boundary conditions and some additional constraints on the states and controls. The differential equations are written here as

$$
\dot{x}=f(t, x, u),
$$

where $x$ is an $n \times 1$ vector of states, $f$ is an $n \times 1$ vector of continuous differential functions, $t$ is the time and $u$ is an $m \times 1$ vector of controls. The states are subject to prescribed initial conditions $x(0)=x_{0}$ and final boundary conditions $\psi\left(t_{f}, x_{f}\right)=0$, where $\psi$ is a $p \times 1$ vector of functions, $t_{0}$ is the initial time and the terminal time $t_{f}$ maybe fixed or free. Some problems also include extra path constraints, which are functions of the state and control variables formulated as

$$
g(t, x, u) \leq 0,
$$

where $g$ is a $q \times 1$ vector. In this paper, we consider problems of the Bolza [22] type, which are focused on minimizing a scalar performance index of the form

$$
J=\phi\left(t_{f}, x_{f}\right)+\int_{t_{0}}^{t_{f}} L(t, x, u) d t
$$

where $\phi$ is a scalar function of the final time $t_{f}$ and final state variables and $L(t, x, u)$ is a scalar function of the time, state $x$ and control $u$.

\section{Direct Collocation and Nonlinear Programming}

\subsection{Haar Discretization Method}

In the discussion of Haar wavelets, we have already addressed how to approximate a function via Haar wavelets and its corresponding operational integration matrix. We 
are expecting to apply this methodology in optimal control problems so that Haar discretization is used in direct collocation. Thus, the continuous solution to a problem will be represented by state and control variables in terms of Haar series and its operational matrix to satisfy the differential equations. The standard interval considered here is denoted as $\tau \in[0,1)$ with collocation points $\tau_{k}$ set as

$$
\tau_{k}=(k-0.5) / M, \quad k=1, \ldots, M,
$$

where $M$ is the number of nodes used in the discretization and also is the maximum wavelet index number. Note that the magnitude of $M$ is in the power of 2, so that the number of collocation points is also increasing in that power. All the collocation points are equally distributed over the entire time interval $[0,1)$ with $\frac{1}{M}$ as the time distance to adjacent nodes. We assume that the derivative of the state variables $\dot{x}(\tau)$ and control variables $u(\tau)$ can be approximated by Haar wavelets with $M$ collocation points, i.e.,

$$
\begin{aligned}
& \dot{x}(\tau) \approx C_{x}^{T} \Psi_{M}(\tau), \\
& u(\tau) \approx C_{u}^{T} \Psi_{M}(\tau),
\end{aligned}
$$

where

$$
C_{x}^{T}=\left[C_{x 1}, C_{x 2}, \ldots, C_{x M}\right], \quad C_{u}^{T}=\left[C_{u 1}, C_{u 2}, \ldots, C_{u M}\right] .
$$

By using the operational integration matrix $P$ defined in (15), the state variables $x(\tau)$ can be expressed as

$$
x(\tau)=\int_{0}^{\tau} \dot{x}\left(\tau^{\prime}\right) d \tau^{\prime}+x_{0}=\int_{0}^{\tau} C_{x}^{T} \Psi_{M}\left(\tau^{\prime}\right) d \tau^{\prime}+x_{0}=C_{x}^{T} P \Psi_{M}(\tau)+x_{0} .
$$

As stated in (11), the expansion of the matrix $\Psi_{M}(\tau)$ at the $M$ collocation points will yield the $M \times M$ Haar matrix $H=\left[h_{1}, h_{2}, \ldots, h_{M}\right]$. It follows that

$$
\dot{x}\left(\tau_{k}\right)=C_{x}^{T} h_{k}, \quad u\left(\tau_{k}\right)=C_{u}^{T} h_{k}, \quad x\left(\tau_{k}\right)=C_{x}^{T} P h_{k}, \quad k=1, \ldots, M .
$$

From the above expression, we can evaluate the variables at any collocation point by using the product of its coefficients vector and the corresponding column vector in the Haar matrix.

\subsection{NLP Solver: SNOPT 6.2}

The NLPS used to solve the NLP problem considered in this work is based on a Sequential Quadrature Programming (SQP) algorithm and is called SNOPT. SNOPT can be used to solve problems like the following: Minimize a performance index $J(x)$, subject to constraints on individual state and/or control variables,

$$
x_{L} \leq x \leq x_{U}
$$

constraints defined by linear combinations of state and/or control variables,

$$
b_{L} \leq A x \leq b_{U}
$$


and/or constraints defined by nonlinear functions of state and/or control variables,

$$
c_{L} \leq c(x) \leq c_{U}
$$

When the Haar collocation method is applied in the optimal control problems, the NLP variables can be set as the unknown coefficients vector of the derivative of the state variables and control variables together with initial and final times, that is,

$$
x=\left[C_{x 1}, C_{x 2}, \ldots, C_{x M}, C_{u 1}, C_{u 2}, \ldots, C_{u M}, t_{0}, t_{f}\right] .
$$

The objective function in (18) is then restated as

$$
J=\phi\left(t_{f}, x(M)\right)+\left(t_{f}-t_{0}\right) \int_{0}^{1} L\left(\left(C_{x}^{T} P \Psi_{M}(\tau)+x_{0}\right), C_{u}^{T} \Psi_{M}(\tau), \tau\right) d \tau .
$$

Since the Haar wavelets are expected to be constant steps at each time interval, the above equation can be simplified as

$$
J=\phi\left(t_{f}, x(M)\right)+\frac{\left(t_{f}-t_{0}\right)}{M} \sum_{k=1}^{M} L\left(\left(C_{x}^{T} P \Psi_{M}\left(\tau_{k}\right)+x_{0}\right), C_{u}^{T} \Psi_{M}\left(\tau_{k}\right), \tau_{k}\right),
$$

with path constraints formulated as

$$
g\left(\left(C_{x}^{T} P \Psi_{M}\left(\tau_{k}\right)+x_{0}\right), C_{u}^{T} \Psi_{M}\left(\tau_{k}\right), \tau_{k}\right) \leq 0 .
$$

Substituting $\dot{x}, u$ and $x$ in (16) with the Haar wavelets expression (24) separately yields

$$
C_{x}^{T} \Psi_{M}\left(\tau_{k}\right)=\left(t_{f}-t_{0}\right) f\left(\tau_{k},\left(C_{x}^{T} P \Psi_{M}\left(\tau_{k}\right)+x_{0}\right), C_{u}^{T} \Psi_{M}\left(\tau_{k}\right)\right)
$$

The system equation constraints and path constraints are all treated as nonlinear constraints in NLP solver. The boundary constraints need to be paid more attention. Since the first and last collocation points are not set as the initial and final time, the initial and final state variables are calculated according to

$$
\begin{gathered}
x_{0}=x(1)-\dot{x}(1) / 2 M, \\
x_{f}=x(M)+\dot{x}(M) / 2 M .
\end{gathered}
$$

In this way, the optimal control problems are transformed into NLP problems in a structured form.

\section{Examples}

In this section, we consider two optimal control problems that have known solution and see how well the results of the NLP with direct collocation can approximate the exact solution. 


\subsection{Brachistochrone Problem}

The brachistochrone problem is that of finding the shape of the curve down which a weight, classically "a bead", acted upon by the force of gravity, will descend from rest and accelerate to a desired point in the least time assuming that there is no friction and the ground force is uniform. Mathematically, this problem is described as finding the minimum time $t_{f}$ for a bead from the starting point $\left[x_{0}, y_{0}\right]$ to the final point $\left[x_{f}, y_{f}\right]$ while satisfying the system equation constraints

$$
\begin{aligned}
& \dot{x}=\sqrt{2 g\left(y_{0}-y\right)} \cos u, \\
& \dot{y}=\sqrt{2 g\left(y_{0}-y\right)} \sin u,
\end{aligned}
$$

where the angle $u$ is the slope of the curve and is treated as control variable of this problem. Using the Haar wavelets collocation method with $M$ collocation points, the objective is to minimize $J=t_{f}$ with system equation constraints

$$
\begin{aligned}
& C_{x} h_{k}=t_{f} \sqrt{2 g\left(y_{0}-\left(C_{y} P h_{x}+y_{0}\right)\right)} \cos \left(C_{u} h_{k}\right), \\
& C_{y} h_{k}=t_{f} \sqrt{2 g\left(y_{0}-\left(C_{y} P h_{x}+y_{0}\right)\right)} \sin \left(C_{u} h_{k}\right), \quad k=1, \ldots, M,
\end{aligned}
$$

and boundary constraints

$$
\begin{array}{cl}
x_{0}=C_{x} P h_{1}+x_{0}-C_{x} h_{1} / 2 M, & y_{0}=C_{y} P h_{1}+y_{0}-C_{y} h_{1} / 2 M, \\
x_{f}=C_{x} P h_{M}+x_{0}-C_{x} h_{M} / 2 M, & y_{f}=C_{y} P h_{M}+y_{0}-C_{y} h_{M} / 2 M,
\end{array}
$$

where $C_{x}, C_{y}, C_{u}$ and $t_{f}$ are all NLP variables solved at the collocation points. The analytical solution for this problem is expressed as a cycloid generated by a circle of diameter $R$ that rolls through angle $\theta$ from the vertical and described mathematically by

$$
\begin{aligned}
& x(\theta)=R(\theta-\sin \theta), \\
& y(\theta)=R(1-\cos \theta) .
\end{aligned}
$$

The analytical optimal control, expressed in terms of time, is

$$
u(t)=\frac{t}{2} \sqrt{g / R}-\frac{\pi}{2} .
$$

For the numerical solution, we assume the starting point is $[0,0]$ and the ending point is $[10,-5]$, use 16 and 32 collocation points separately for the Haar wavelets discretization method. The initial guess of the solution is the straight line connecting the starting and ending points. Therefore, the initial control and NLP variables are estimated according to this coarse initial guess. The trajectory and control history results using this new method is shown in Figs. 2 and 3, separately, and compared to the analytical results. Also, there are results of using the Chebyshev pseudospectral (CP) collocation method to solve the same problem with the same number of collocation points. 
Fig. 2 Trajectory for brachistochrone problem
Fig. 3 Time history of control for brachistochrone problem
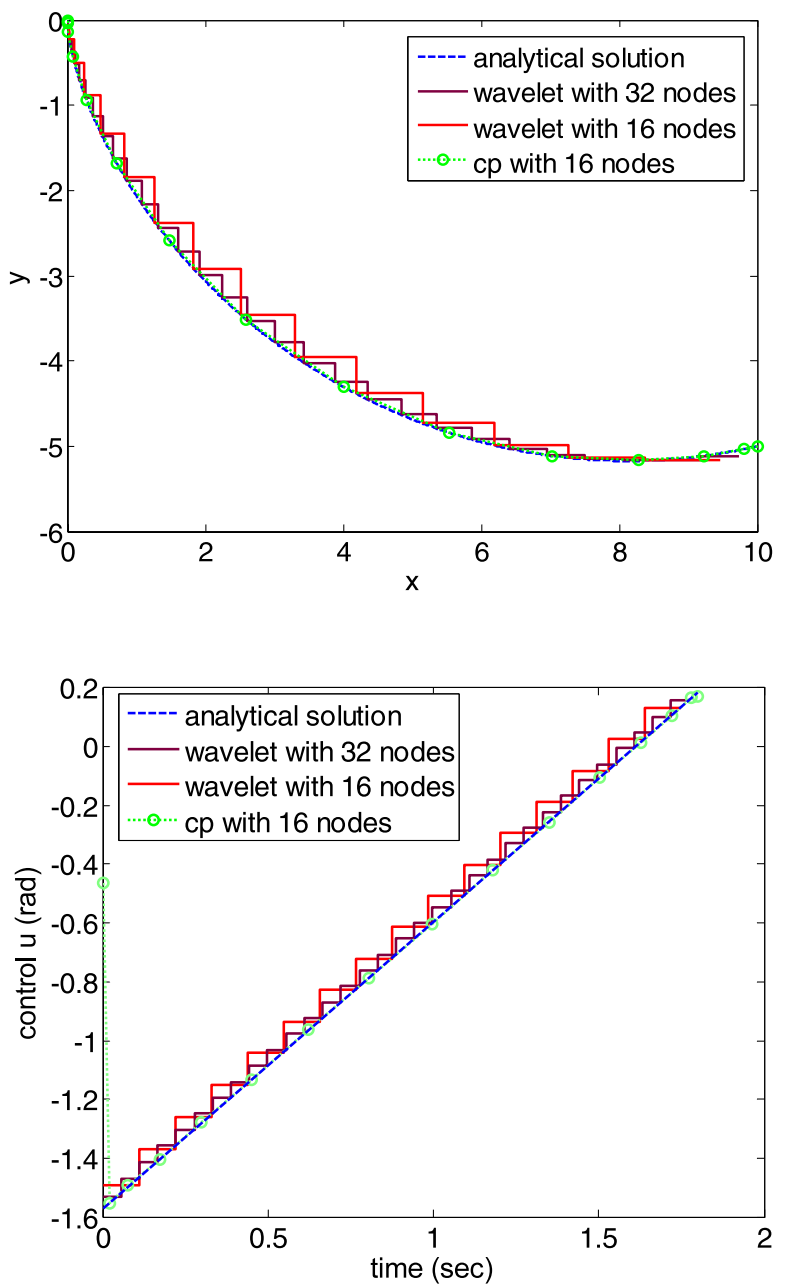

From Figs. 2 and 3, it can be seen that the results generated by the Haar wavelets collocation method made good approximation to the analytical solution. When the number of collocation points increases, the accuracy is improved and further increase will make the final trajectory converge to the exact solution. The absolute error of the objective function between wavelet solution $J$ and analytical solution $J^{*}$ with respect to the number of nodes is shown in Fig. 4.

\subsection{Discrete Optimal Control Problem}

The discrete optimal control problem considered here is to use bang-bang control with maximum and minimum bounds so as to minimize the cost function

$$
J=t_{f},
$$


Fig. 4 Objective function error for brachistochrone problem

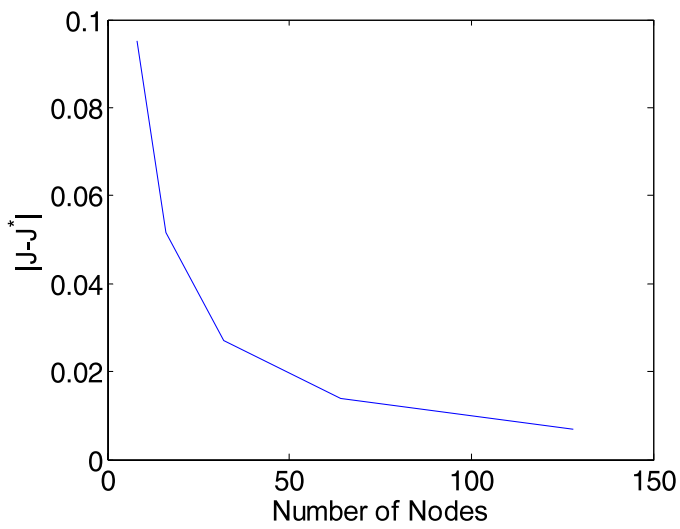

subject to simple system equation constraints

$$
\begin{aligned}
& \dot{x}_{1}=x_{2}, \\
& \dot{x}_{2}=u
\end{aligned}
$$

and initial state variable constraints

$$
\begin{array}{ll}
x_{1}(0)=x_{10}=1, & x_{2}(0)=x_{20}=3, \\
x_{1}\left(t_{f}\right)=0, & x_{2}\left(t_{f}\right)=0,
\end{array}
$$

where $u$ is the control and is constrained so that $|u(t)| \leq 1$. The analytical solution for this problem is [16]

$$
\begin{aligned}
& x_{1}(t)= \begin{cases}-t^{2} / 2+x_{20} \cdot t+x_{10}, & t<t_{1}, \\
t^{2} / 2-t_{f} \cdot t+t_{f}^{2} / 2, & t>t_{1},\end{cases} \\
& x_{2}(t)= \begin{cases}-t+x_{20}, & t<t_{1}, \\
t-t_{f}, & t>t_{1},\end{cases} \\
& u(t)= \begin{cases}-1, & t<t_{1}, \\
1, & t>t_{1},\end{cases}
\end{aligned}
$$

where the switching time is calculated at $t_{1}=x_{20}+\sqrt{0.5 x_{20}^{2}+x_{10}}$ and the optimized final time $t_{f}=2 t_{1}-x_{20}$. For the DCNLP solutions, we assume that the trajectory starts from [1,3] and ends at [0,0], again use 16 and 32 nodes in Haar wavelets collocation and the initial guess of the state variables are straight line connecting the starting and final points. Then the results to analytical and CP methods are compared in Fig. 5 for state variables and Fig. 6 for controls.

It's obvious to see that Haar wavelet collocation has an advantage in bang-bang optimal control problems when the switching time is unknown. Instead of the slope solution with long and unsteady time interval before and after the switching point in CP method, the wavelet solution provides bang-bang control wave which switch values very close to the exact analytical switching time. The convergence rate for the 
Fig. 5 Time history of states for bang-bang problem
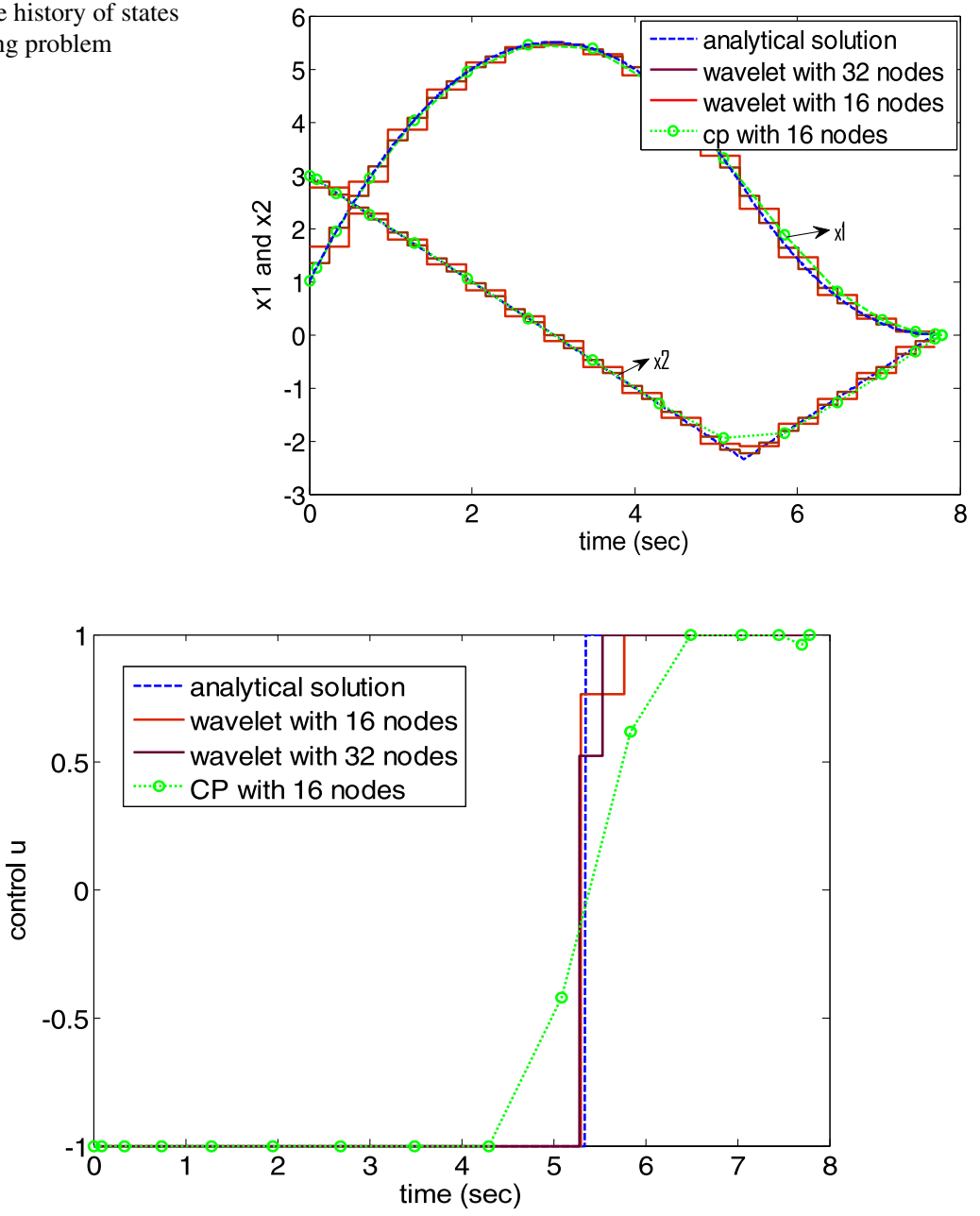

Fig. 6 Time history of control for bang-bang problem

wavelet solution is shown in Fig. 7. When the number of nodes increases, it is expected that the wavelet solution will generate the optimal control solution with error close to zero. Besides, the state variables of the wavelet converge to the analytical solution.

\section{Conclusions}

A new collocation method is applied to solve two well-known optimal control problems using a nonlinear programming solver. The system equations are all expressed via Haar wavelets with unknown coefficients and solving those unknown coefficients while optimizing the performance index becomes the task of this kind of parameter- 


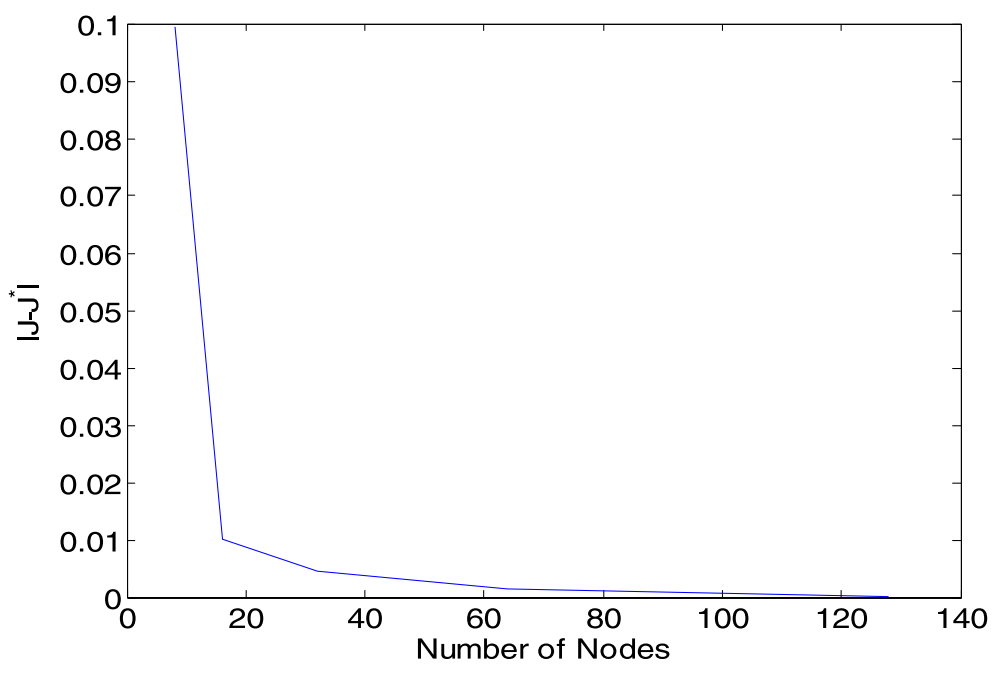

Fig. 7 Objective function error for bang-bang problem

ized problems. This new method produces results similar to other collocation methods for the continuous optimal control problem and shows advantages in discrete optimal control problems when the switching time is unknown.

Open Access This article is distributed under the terms of the Creative Commons Attribution Noncommercial License which permits any noncommercial use, distribution, and reproduction in any medium, provided the original author(s) and source are credited.

\section{References}

1. Boggess, A., Narcowich, F.J.: A First Course in Wavelets with Fourier Analysis. Prentice-Hall, Englewood Cliffs (2001)

2. Bertoluzza, S., Naldi, G.: A wavelet collocation method for the numerical solution of partial differential equations. Appl. Comput. Harmon. Anal. 3(1), 1-9 (1996)

3. Lepik, U.: Numerical solution of differential equations using Haar wavelets. Math. Comput. Simul. 68, 127-143 (2004)

4. Beylkin, G.: On wavelet-based algorithms for solving differential equations. In: Wavelets: Mathematics and Applications, pp. 449-466. CRC Press, Boca Raton (1994)

5. Xu, J.C., Shann, W.C.: Galerkin-wavelet methods for two-point boundary value problems. Numer. Math. 63, 123-144 (1992)

6. Chen, C.F., Hsiao, C.H.: Wavelet approach to optimizing dynamic systems. IEE Proc. Control Theory Appl. 146(2), 213-219 (1999)

7. Karimi, H.R., Maralani, P.J., Moshiri, B., Lohmann, B.: Haar wavelet-based control of time-varying state-delayed systems: a computational method. Int. J. Comput. Math. 83(2), 235-246 (2006)

8. Razzaghi, M., Yousefi, S.: Legendre wavelets method for constrained optimal control problems. Math. Methods Appl. Sci. 25, 529-539 (2002)

9. Hsiao, C.H., Wang, J.W.: Optimal control of linear time-varying systems via Haar wavelets. J. Optim. Theory Appl. 103(3), 641-655 (1999)

10. Holmstrom, K., Goran, A.O., Edvall, M.M.: User's Guide for TOMLAB /SNOPT. Tomlab Optimization Inc. (2005). http://tomlab.biz

11. Gano, S.E., Perez, V.M., Renaud, J.E.: Development and verification of a MATLAB driver for the SNOPT optimization software. AIAA Paper 2001-1620 (2001) 
12. Hargraves, C.R., Paris, S.W.: Direct trajectory optimization using nonlinear programming and collocation. J. Guid. Control Dyn. 10(4), 338-342 (1987)

13. Betts, J.T., Huffman, W.P.: Path-constrained trajectory optimization using sparse sequential quadratic programming. J. Guid. Control Dyn. 16(1), 59-68 (1993)

14. Fahroo, M., Ross, I.M.: Direct trajectory optimization by a Chebyshev pseudospectral method. J. Guid. Control Dyn. 25(1), 160-166 (2002)

15. Pietz, J.A.: Pseudospectral collocation methods for the direct transcription of optimal control problems. M.A. Thesis, Dept. of Computational and Applied Mathematics, Rice University, Houston, TX (2003)

16. Benson, D.: A Gauss pseudospectral transcription for optimal control. Ph.D. Thesis, Department of Aeronautics and Astronautics, MIT (Nov. 2004)

17. Huntington, G.T., Benson, D.A., Rao, A.V.: A comparison of accuracy and computational efficiency of three pseudospectral methods. In: Proc. of the AIAA Guidance, Navigation, and Control Conference, Hilton Head, SC, August 21-24, 2007

18. Horie, K., Conway, B.A.: Optimal, aeroassisted orbital interception. J. Guid. Control Dyn. 22(5), 625-631 (1999)

19. Geiger, B.R., Horn, J.F., DeLullo, A.M., Long, L.N.: Optimal path planning of UAVs using direct collocation with nonlinear programming. In: AIAA Guidance, Navigation, and Control Conference and Exhibit, Keystone, Colorado. AIAA 2006-6199 (2006)

20. Herman, A.L., Spencer, D.B.: Optimal, low-thrust earth-orbit transfers using higher-order collocation methods. J. Guid. Control Dyn. 25(1), 40-47 (2002)

21. Gu, J.S., Jiang, W.S.: The Haar wavelets operational matrix of integration. Int J. Syst. Sci. 27(7), 623-628 (1996)

22. Bryson, A.E., Ho, Y.C.: Applied Optimal Control, Optimization, Estimation and Control. Hemisphere, Washington (1975) 